\title{
LINEAR AND NONLINEAR BOUNDARY PROBLEMS FOR HARMONIC FUNCTIONS ${ }^{1}$
}

\author{
M. H. MARTIN
}

1. Introduction. In this paper we construct integral identities to obtain results on the uniqueness of solutions to linear and nonlinear boundary problems. As has been pointed out, questions of uniqueness are important in hydrodynamics [1] and can be more difficult than questions of existence [11]. In the main our attention will be concentrated on the following problem.

What can be said about the uniqueness of a function $u$, harmonic in a region $S$, if along the boundary $C$ of $S$ the external normal derivative $u_{n}$ is a prescribed separable function

$$
u_{n}=h(s) f(u)
$$

of $u$ and the arc length $s$ of $C$ ?

For simplicity $S$ is assumed simply connected and its boundary $C$ to consist of a single analytic curve. The functions $h(s), f(u)$ are analytic in their arguments for all values occurring; $u$ is supposed to have continuous partial derivatives of the first order in $S+C$ and, according to a theorem of Lewy [12], is analytically extensible across $C$. Thus we may assume that $S$ lies in the interior of a region $R$ within which $u$ is harmonic.

If $f$ is a constant, the problem becomes Neumann's problem [2], as the values of $u_{n}$ are now prescribed on $C$. Here the uniqueness question is settled by the integral identity

$$
\int_{C} u u_{n} d s=\int_{S}\left(u_{x}^{2}+u_{y}^{2}+u \Delta u\right) d S,
$$

for, as Gauss [3] himself observed, this identity implies that $u$ must be constant if its normal derivative vanishes on $C$, and consequently two harmonic functions differ only by a constant, if their normal derivatives are equal on $C$.

If $f=u$, we have the so-called Problem of Steklov [4]

$$
u_{n}=h(s) u \text {. }
$$

This problem arises in several areas of mathematical physics; for

Presented to the Society, August 29, 1958; received by the editors August 2, 1958.

1 This work was supported by the Office of Ordnance Research, U. S. Army, under Contract DA-36-034-ORD-1486 with the University of Maryland. 
$h(s)<0$ the problem was treated by Fourier in his theory of heat [5]; for $h(s)>0$ the problem arises in the linearized theory of water waves [6] and in Weinstein's [7] theory of jets. For this problem our method constructs the integral identity

$$
\begin{aligned}
\int_{C} \lambda\left(u_{2} \frac{\partial u_{1}}{\partial n}-u_{1} \frac{\partial u_{2}}{\partial n}\right) d s \\
\quad=\int_{S}\left[u_{2}^{2}\left(\lambda_{x}^{2}+\lambda_{y}^{2}\right)+\lambda\left(u_{2} \Delta u_{1}-u_{1} \Delta u_{2}\right)\right] d S, \quad \lambda=u_{1} / u_{2},
\end{aligned}
$$

valid for any pair of functions $u_{1}, u_{2}$ of class $C^{(2)}$ in $R$, provided their ratio $\lambda$ has continuous partial derivatives of the first order in $R$. The identity itself is readily verified as an application of Gauss's theorem, and in $\$ 3$ we use it to derive a uniqueness theorem for solutions to the Steklov problem. We shall also see that Weinstein's local uniqueness theorem (in the restricted sense) [7], so important in the theory of jets, may, in the form given it by Friedrichs [8], be derived from this identity.

In $\$ 4$ we obtain a uniqueness theorem for the nonlinear problem

$$
u_{n}=h(s) u^{1+p}, \quad p=\text { const., }
$$

in case $p$ is an even, positive integer. That such problems have solutions is confirmed by an example.

2. The integral identities. Our starting point is Gauss's theorem

$$
\int_{C}\left(A x_{n}+B y_{n}\right) d s=\int_{S}\left(A_{x}+B_{y}\right) d S
$$

applied to a simply connected region $S$ bounded by a simple closed curve $C$ consisting of finitely many smooth arcs all lying in an open region $R$ within which the functions $A, B$ have continuous partial derivatives of the first order with respect to $x, y$.

Specifically we take

$$
A=A\left(u_{1}, p_{1}, q_{1}, u_{2}, p_{2}, q_{2}\right), \quad B=B\left(u_{1}, p_{1}, q_{1}, u_{2}, p_{2}, q_{2}\right)
$$

where $u_{1}, u_{2}$ are two functions of $x, y$ having continuous partial derivatives of the second order in $R$, and, following Monge, we write

$$
p_{i}=\frac{\partial u_{i}}{\partial x}, \quad q_{i}=\frac{\partial u_{i}}{\partial y}, \quad r_{i}=\frac{\partial^{2} u_{i}}{\partial x^{2}}, \quad s_{i}=\frac{\partial^{2} u_{i}}{\partial x \partial y}, \quad t_{i}=\frac{\partial^{2} u_{i}}{\partial y^{2}},
$$

One finds without difficulty that

$$
(i=1,2) \text {. }
$$




$$
\begin{aligned}
\int_{C}\left(A x_{n}+B y_{n}\right) & d s \\
= & \int_{S}\left(A_{u_{i}} p_{i}+B_{u_{i}} q_{i}+A_{p_{i}} r_{i}+\left(A_{q_{i}}+B_{p_{i}}\right) s_{i}+B_{q_{i}} t_{i}\right) d S,
\end{aligned}
$$

the usual summation convention for repeated indices being understood for $i=1,2$. If we assume that the functions $A, B$ satisfy CauchyRiemann equations

$$
A_{p_{i}}=B_{q_{i}}, \quad A_{q_{i}}=-B_{p_{i}} \quad(i=1,2),
$$

in the variables $p_{i}, q_{i}$, we obtain

$$
\int_{C}\left(A x_{n}+B y_{n}\right) d s=\int_{S}\left(A_{u_{i}} p_{i}+B_{u_{i}} q_{i}+A_{p_{i}} \Delta u_{i}\right) d S .
$$

Let us specialize the functions $A, B$ further by assuming

$$
A=\alpha_{i} p_{i}, \quad B=\alpha_{i} q_{i}, \quad \alpha_{i}=\alpha_{i}\left(u_{1}, u_{2}\right),
$$

to obtain the identity

$$
\int_{C} \alpha_{i} \frac{\partial u_{i}}{\partial n} d s=\int_{S}\left(\frac{\partial \alpha_{i}}{\partial u_{j}} p_{i} p_{j}+\frac{\partial \alpha_{i}}{\partial u_{j}} q_{i} q_{j}+\alpha_{i} \Delta u_{i}\right) d S,
$$

$$
(i, j=1,2) \text {, }
$$

upon which all further considerations are based.

As a simple illustration of the use of this identity, set

$$
\alpha_{1}=u_{1}-u_{2}, \quad \alpha_{2}=u_{2}-u_{1} .
$$

One finds

$$
\begin{aligned}
\int_{C}\left(u_{1}-u_{2}\right) & \left(\frac{\partial u_{1}}{\partial n}-\frac{\partial u_{2}}{\partial n}\right) d s \\
& =\int_{S}\left[\left(p_{1}-p_{2}\right)^{2}+\left(q_{1}-q_{2}\right)^{2}+\left(u_{1}-u_{2}\right)\left(\Delta u_{1}-\Delta u_{2}\right)\right] d S .
\end{aligned}
$$

With a view to later developments, observe that the quadratic forms in the $p_{i}, q_{i}$ appearing in (2.1) have become perfect squares.

This reduces to the integral identity (1.1), associated with the Neumann problem if we replace $u=u_{1}-u_{2}$.

In order that the quadratic forms in the fundamental identity (2.1) be perfect squares, we require that $\alpha_{1}, \alpha_{2}$ satisfy the condition 


$$
\left(\frac{\partial \alpha_{1}}{\partial u_{2}}+\frac{\partial \alpha_{2}}{\partial u_{1}}\right)^{2}-4 \frac{\partial \alpha_{1}}{\partial u_{1}} \frac{\partial \alpha_{2}}{\partial u_{2}}=0 .
$$

If we search for functions $\alpha_{i}$ of the form

$$
\alpha_{1}=u_{2} \sigma\left(u_{1}, u_{2}\right), \quad \alpha_{2}=-u_{1} \sigma\left(u_{1}, u_{2}\right),
$$

this condition reduces to

$$
\left(u_{1} \sigma_{u_{1}}+u_{2} \sigma_{u_{2}}\right)^{2}=0 .
$$

This means that $\sigma$ is a function $\sigma(\lambda)$ of the ratio $\lambda=u_{1} / u_{2}$ alone, and if we denote differentiation by a prime, the fundamental identity yields

$$
\begin{aligned}
\int_{C} \sigma\left(u_{2} \frac{\partial u_{1}}{\partial n}-u_{1} \frac{\partial u_{2}}{\partial n}\right) d s \\
=\int_{S}\left[\sigma^{\prime}\left(p_{1}-\lambda p_{2}\right)^{2}+\sigma^{\prime}\left(q_{1}-\lambda q_{2}\right)^{2}+\sigma\left(u_{2} \Delta u_{1}-u_{1} \Delta u_{2}\right)\right] d S,
\end{aligned}
$$

which reduces to (1.2) when $\sigma=\lambda$.

If we seek to satisfy (2.2) with

$$
\alpha_{1}=\frac{\sigma\left(u_{1}, u_{2}\right)}{u_{1}^{1+p}}, \quad \alpha_{2}=-\frac{\sigma\left(u_{1}, u_{2}\right)}{u_{2}^{1+p}}, \quad p=\text { const. },
$$

we obtain a partial differential equation

$$
\begin{aligned}
& \left(u_{1}^{1+p} \sigma_{u_{1}}+u_{2}^{1+p} \sigma_{u_{2}}\right)^{2}-4(1+p) u_{1}^{p} u_{2}^{p} \sigma\left(u_{1} \sigma_{u_{1}}\right. \\
& \left.+u_{2} \sigma_{u_{2}}\right) \\
& +4(1+p)^{2} u_{1}^{p u_{2}^{p} \sigma^{2}}=0
\end{aligned}
$$

for the unknown function $\sigma$. This simplifies to

$$
\sigma_{\phi}^{2}-4(1+p) \rho \sigma \sigma_{\rho}+4(1+p)^{2} \sigma^{2}=0,
$$

if we introduce "polar coordinates" $\rho, \phi$ in the $\left(u_{1}, u_{2}\right)$-plane by placing

$$
u_{1}=\rho \operatorname{sech}^{2 / p} \frac{p}{2} \phi, \quad u_{2}=\rho \operatorname{csch}^{2 / p} \frac{p}{2} \phi .
$$

Formulas for the inverse transformation will be needed, and they are

$$
\frac{1}{\rho^{p}}=\frac{1}{u_{1}^{p}}-\frac{1}{u_{2}^{p}}, \quad \tanh \frac{p}{2} \phi=\left(\frac{u_{1}}{u_{2}}\right)^{p / 2} .
$$

Choosing the solution $\sigma=\rho^{1+p}$ of (2.3), we find 


$$
\alpha_{1}=\left(\frac{\rho}{u_{1}}\right)^{1+p}=\rho_{u_{1}}, \quad \alpha_{2}=-\left(\frac{\rho}{u_{2}}\right)^{1+p}=\rho_{u_{2}},
$$

and (2.1) yields

$$
\int_{C} \rho_{n} d s=\int_{S} \Delta \rho d S
$$

where, of course,

$$
\begin{aligned}
\rho_{n} & =\left(\frac{\rho}{u_{1}}\right)^{1+p} \frac{\partial u_{1}}{\partial n}-\left(\frac{\rho}{u_{2}}\right)^{1+p} \frac{\partial u_{2}}{\partial n}, \\
\Delta \rho & =\rho_{u_{i} u_{j}} p_{i} p_{j}+\rho_{u_{i} u} q_{i} q_{j}+\rho_{u_{i}} \Delta u_{i} .
\end{aligned}
$$

The latter equation holds some interesting consequences. Since the quadratic forms are perfect squares

$$
\rho_{u_{1} u_{1}} \rho_{u_{2} u_{2}}-\stackrel{\rho_{u_{1} u_{2}}^{2}}{=}=0,
$$

and $\rho=\rho\left(u_{1}, u_{2}\right)$ is accordingly a developable surface. One verifies, by direct calculation from (2.4) that

$$
\Delta \rho=(1+p) \rho\left(\phi_{x}^{2}+\phi_{y}^{2}\right)+\rho_{u_{i}} \Delta u_{i},
$$

and (2.5) becomes

$$
\int_{C} \rho_{n} d s=\int_{S}\left[(1+p) \rho\left(\phi_{x}^{2}+\phi_{y}^{2}\right)+\rho_{u_{i}} \Delta u_{i}\right] d S .
$$

If we place $u=\rho$ in (1.1) we arrive at the identity

$$
\int_{C} \rho \rho_{n} d s=\int_{S}\left[\rho_{x}^{2}+\rho_{y}^{2}+(1+p) \rho^{2}\left(\phi_{x}^{2}+\phi_{y}^{2}\right)+\rho \rho_{u_{i}} \Delta u_{i}\right] d S
$$

upon which we base the uniqueness theorem of $\$ 4$.

3. The Steklov problem. In this boundary problem

$$
\Delta u=0 \text { in } S, \quad u_{n}=h(s) u \text { on } C .
$$

The solution is unique if $h$ is always negative; but need not be unique if $h$ is positive.

Indeed, following Carleman [9], we can show that even the solution to the more general problem

$$
\Delta u=0 \text { in } S, \quad u_{n}=f(u, s) \text { on } C,
$$


is unique, if $f_{u}$ is always negative. If this problem should possess two solutions $u_{1}, u_{2}$, take $u=u_{1}-u_{2}$ in (1.1) and obtain

$$
\int_{C} f_{u}(\xi, s) u^{2} d s-\int_{S}\left(u_{x}^{2}+u_{y}^{2}\right) d S=0,
$$

where $\xi$ is some value between $u_{1}, u_{2}$. Since $f_{u}<0$, this implies $u=0$, and uniqueness as stated.

If $h>0$ the solution need not be unique, as is illustrated by the harmonic functions

$$
u=A r^{h} \cos h \theta+B r^{h} \sin h \theta, \quad h=1,2, \cdots .
$$

Here $A, B$ are arbitrary constants and all these functions $u$ solve the Steklov problem for the unit circle $r=1$.

On the other hand Boggio [10] and Weinstein [11] have pointed out that there are no other solutions to this problem. To see this, note that the function $r u_{r}-h u$ is harmonic and vanishes on the unit circle. Thus it vanishes in the interior and we may place $r u_{r}=h u$ in Laplace's equation $r\left(r u_{r}\right)_{r}+u_{\theta \theta}=0$. This yields $u_{\theta \theta}+h^{2} u=0$, and therefore $u=F_{1}(r) \cos h \theta+F_{2}(r) \sin h \theta$. For $u$ to be harmonic $F_{1}, F_{2}$ must satisfy the ordinary differential equation $r\left(r F^{\prime}\right)^{\prime}-h^{2} F=0$, for which the only regular solutions are proportional to $r^{h}$.

The following theorem is an immediate consequence of the identity (1.2).

THEOREM. If $u_{2} \not \equiv 0$ is a solution of the boundary problem

$$
\Delta u=0 \text { in } S, \quad u_{n}=h(s) u \text { on } C,
$$

harmonic in $R \supset S$, any other such solution $u_{1}$ for which the ratio $\lambda=u_{1} / u_{2}$ is regular in $R$, is linearly dependent on $u_{2}$.

As M. Riesz has pointed out, the ratio $\lambda$ will be regular whenever the nodal lines of $u_{2}$ are also nodal lines of $u_{1}$.

Indeed, under the conditions stated in the theorem, (1.2) yields

$$
\int_{C} \lambda\left(u_{2} \frac{\partial u_{1}}{\partial n}-u_{1} \frac{\partial u_{2}}{\partial n}\right) d s=\int_{S} u_{2}^{2}\left(\lambda_{x}^{2}+\lambda_{y}^{2}\right) d S, \quad \lambda=u_{1} / u_{2},
$$

from which $\lambda=$ const., obviously.

As my colleague J. B. Diaz has kindly observed, the same result holds for two solutions of the equation

$$
\Delta u+c u=0, \quad c=c(x, y),
$$

for the vibrating membrane. 
The following theorem, due to Weinstein [7], plays an important role in the theory of jets.

Local UNIQUeNESS TheOREM. If $u$, harmonic in $S$, vanishes on a part $C_{1}$ of $C$ and if $u_{n}=h(s) u$ holds on the remaining part $C_{2}$ of $C$, then $u$ vanishes identically in $S+C$.

Friedrichs [8] pointed out, with the help of Jacobi's multiplicative variation, that the theorem holds, if a harmonic function $u_{2}$ which does not vanish in $S+C$ can be found for which

$$
\frac{\partial u_{2}}{\partial n}=h(s) u_{2} \text { on } C_{2} \text {. }
$$

Friedrichs' result is an immediate consequence of (3.1), if we take $u_{1}=u$. Clearly the integrand in the line integral around $C$ vanishes, for $\lambda$ vanishes on $C_{1}$ and the other factor vanishes on $C_{2}$. Thus $\lambda=$ const., and since $u_{1}=\lambda u_{2}$ vanishes on $C_{1}$ while $u_{2}$ does not, we conclude $\lambda=0$.

4. A nonlinear boundary problem. As an example of a nonlinear boundary problem, consider

$$
\Delta u=0 \text { in } S, \quad u_{n}=h(s) u^{1+p} \text { on } C .
$$

For $p=3$ and $h(s)<0$, this is the Stefan-Boltzman radiation problem, for which uniqueness was established by Carleman [9]. Our treatment of the uniqueness question disregards the sign of $h(s)$ and is based on the formula

$$
\int_{C} \rho \rho_{n} d s=\int_{S}\left[\rho_{x}^{2}+\rho_{y}^{2}+(1+p) \rho^{2}\left(\phi_{x}^{2}+\phi_{y}^{2}\right)\right] d S,
$$

which arises from (2.7) when $u_{1}, u_{2}$ are harmonic. For convenience we recall from (2.4) that $\rho, \phi$ are defined by

$$
\frac{1}{\rho^{p}}=\frac{1}{u_{1}^{p}}-\frac{1}{u_{2}^{p}}, \quad \tanh \frac{p}{2} \phi=\left(\frac{u_{1}}{u_{2}}\right)^{p / 2}
$$

and that

$$
\rho_{n}=\left(\frac{\rho}{u_{1}}\right)^{1+p} \frac{\partial u_{1}}{\partial n}-\left(\frac{\rho}{u_{2}}\right)^{1+p} \frac{\partial u_{2}}{\partial n} .
$$

The precise result is contained in the following theorem.

THEOREM. If $u_{2}$ is a nonconstant solution of the boundary problem

$$
\Delta u=0 \text { in } S, \quad u_{n}=h(s) u^{1+p} \text { on } C, \quad(p=2,4, \cdots),
$$


harmonic in $R \supset S$, no other such solution $u_{1}$ exists for which the ratio $\lambda=u_{1} / u_{2}$ is regular in $R$ and $|\lambda|<1$.

Assume that a second solution $u_{1}$ exists as stated. Inasmuch as

$$
\rho=\frac{\lambda}{\left(1-\lambda^{p}\right)^{1 / p}} u_{2}, \quad \phi=\frac{2}{p} \operatorname{arctanh} \lambda^{p / 2},
$$

and $|\lambda|<1$, the functions $\rho, \phi$ have continuous partial derivatives of the first order in $R$. If $u_{1}, u_{2}$ meet the boundary condition given in the theorem, $\rho_{n}=0$, whereupon (4.1) implies that $\rho, \phi$ are both constant. Thus $u_{2}$ would be constant, contrary to hypothesis.

Compared with the linear problem, uniqueness for the solution in the nonlinear problem appears to require an additional hypothesis, namely $|\lambda|<1$. Some stipulation of this nature seems to be necessary to insure uniqueness, for if $u_{1}$ is a solution, $u_{2}=-u_{1}$ is also a solution.

To see that such boundary problems actually have solution, consider the harmonic function

$$
u=\frac{r \cos \theta-a}{a^{2}+r^{2}-2 a r \cos \theta}, \quad r<a .
$$

This is the real part, in polar coordinates $r, \theta$, of the analytic function $1 /(z-a)$, regular save at $z=a$. One finds

$$
u_{r}=\frac{2 a r-\left(a^{2}+r^{2}\right) \cos \theta}{\left(a^{2}+r^{2}-2 a r \cos \theta\right)^{2}},
$$

and therefore that

$u_{r}=h(s) u^{1+p}, \quad h(s)=\frac{\left[2 a r-\left(a^{2}+r^{2}\right) \cos \theta\right]\left[a^{2}+r^{2}-2 a r \cos \theta\right]^{p-1}}{(r \cos \theta-a)^{p+1}}$.

Thus $u$ is a solution of this boundary problem set for the region $S$ interior to the coordinate circle of radius $r$.

\section{BIBLIOGRAPHY}

1. A. Weinstein, On surface waves, Canad. J. Math. vol. 1 (1949) pp. 271-278.

2. C. Neumann, Revision einiger allgemeinen Sätze aus der Theorie des Logarithmischen Potentials, Math. Ann. vol. 3 (1871).

3. W. F. Osgood, Lehrbuch der Funktionentheorie, vol. 1, Berlin, 1920, p. 624.

4. M. W. Steklov, Sur les problèmes fondamentaux de la physique mathématique, Ann. Sci. École Norm. Sup. vol. 19 (1902) pp. 455-490.

R. Weinstock, Inequalities for a classical eigenvalue problem, Tech. Report 37. Contr. N6ori-106 Task Order 5 (NR-043-992) Department of Mathematics, Stanford University.

5. J. B. J. Fourier, The analytic theory of heat, English translation by A. Freeman, 1888 , p. 29 and p. 94. 
6. H. Lamb, Hydrodynamics, Cambridge, 1924, pp. 341-450.

7. A. Weinstein, Ein hydrodynamicher Unitätssatz, Math. Z. vol. 19 (1924) pp. 265-275.

8. K. FRIEDRICHS, Über ein Minimumproblem für Potentialströmungen mit freien Rande, Math. Ann. vol. 109 (1933) in particular pp. 77-81.

9. T. Carleman, Über eine nichtlineare Randwertaufgabe bei der Gleichung $\Delta u=0$, Math. Z. vol. 9 (1921) pp. 35-43.

10. T. Boggio, Sulle funzioni di variabile complessa ui un'area circolare, Rendiconti della R. Accademia di Torino vol. 47 (1912) pp. 22-37.

11. A. Weinstein, Fluid motion with free boundaries, Proceedings of the First Symposium in Applied Mathematics, American Mathematical Society, 1949, pp. $1-18$.

12. H. Lewy, $A$ note on harmonic functions and a hydrodynamical application, Proc. Amer. Math. Soc. vol. 3 (1952) pp. 111-113.

UNIVERSITY OF MARYLAND

\section{FUNCTIONS HAVING POSITIVE REAL PART IN AN ELLIPSE}

W. C. ROYSTER

1. Introduction. Carathéodory [1] proved that if an analytic function $f(z), f(0)=1$, is regular and has positive real part in the unit circle then the $n$th coefficient must lie in the closed circle with center at the origin and with radius equal to 2 , the extremal function being essentially $(1+z)(1-z)^{-1}$. This theorem has been used extensively in the study of various subclasses of univalent functions. It has been generalized to functions which are regular and have positive real part in an annulus by Nehari [2].

In this paper we obtain an analogous theorem for functions which are regular and have positive real part in an ellipse.

2. The main result. Let $f(z)$ be regular in the ellipse $E$ with foci at \pm 1 and semiaxes $a>b, a>1$. Such a function is representable by a series of Tchebychef polynomials which converges uniformly in $E$, $[3 ; 4]$. That is,

$$
f(z)=\sum_{n=0}^{\infty} a_{n} T_{n}(z), \quad z \in E
$$

where $T_{n}(z)=\cos (n \arccos z)$.

$E$ may be represented parametrically by $z=a \cos t+i b \sin t$, $0 \leqq t<2 \pi$. Since $a^{2}-b^{2}=1$ we may write $a=\cosh s, b=\sinh s, s>0$.

Received by the editors January 16, 1958, and in revised form, June 29, 1958. 